I ssayas Tekeste, Begüm Demir

\title{
Advanced Local Binary Patterns for Remote Sensing I mage Retrieval
}

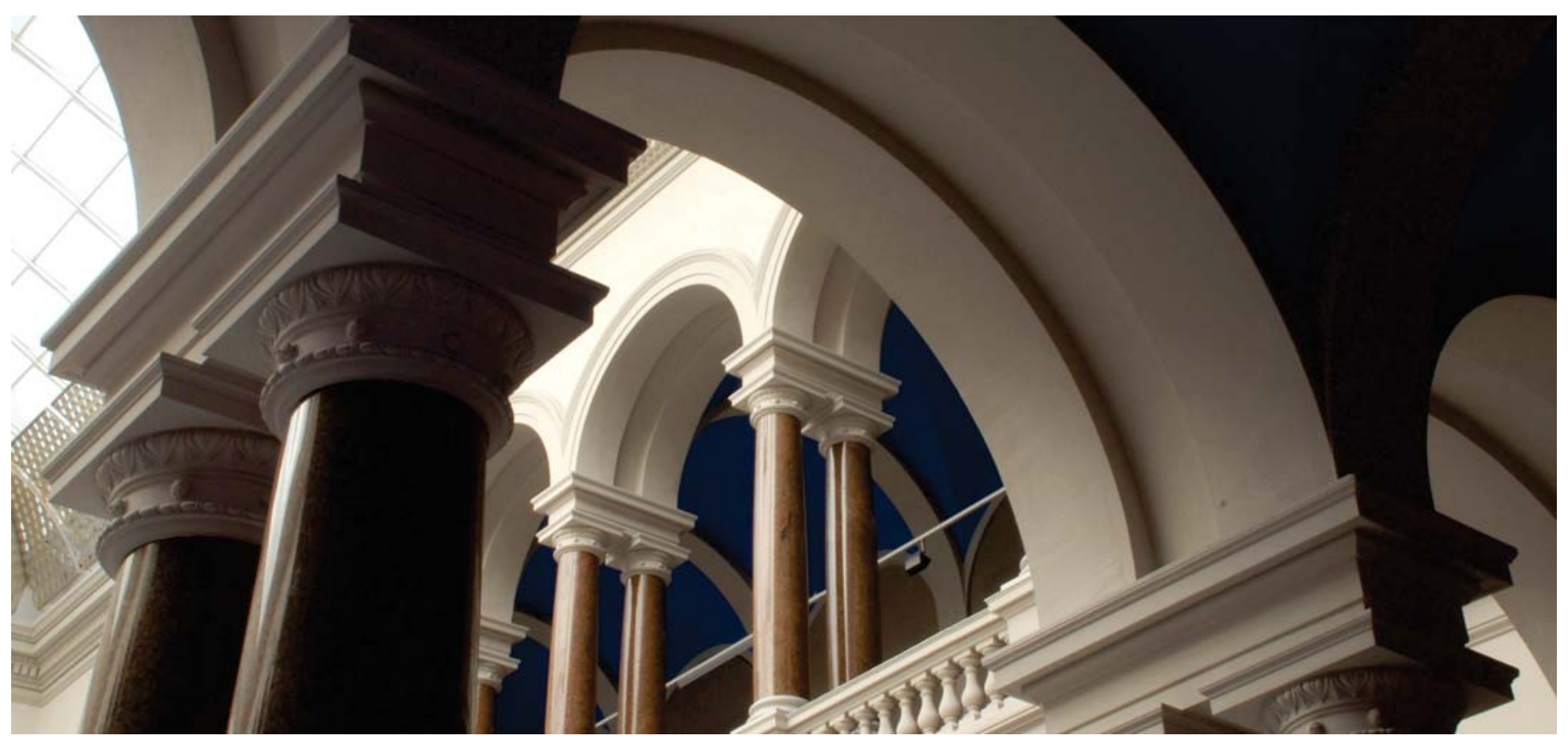

Tekeste, I., \& Demir, B. (2018). Advanced Local Binary Patterns for Remote Sensing Image Retrieval. IGARSS 2018 - 2018 IEEE International Geoscience and Remote Sensing Symposium. pp. 6855-6858. https://doi.org/10.1109/igarss.2018.8518856 


\title{
ADVANCED LOCAL BINARY PATTERNS FOR REMOTE SENSING IMAGE RETRIEVAL
}

\author{
Issayas Tekeste ${ }^{1}$, Begüm Demir ${ }^{2}$ \\ ${ }^{1}$ Dept. of Information Engineering and Computer Science, University of Trento, Italy \\ ${ }^{2}$ Faculty of Electrical Engineering and Computer Science, TU Berlin, Berlin, Germany
}

\begin{abstract}
The standard Local Binary Pattern (LBP) is considered among the most computationally efficient remote sensing (RS) image descriptors in the framework of large-scale content based RS image retrieval (CBIR). However, it has limited discrimination capability for characterizing high dimensional RS images with complex semantic content. There are several LBP variants introduced in computer vision that can be extended to RS CBIR to efficiently overcome the above-mentioned problem. To this end, this paper presents a comparative study in order to analyze and compare advanced LBP variants in RS CBIR domain. We initially introduce a categorization of the LBP variants based on the specific CBIR problems in RS, and analyze the most recent methodological developments associated to each category. All the considered LBP variants are introduced for the first time in the framework of RS image retrieval problems, and have been experimentally compared in terms of their: 1) discrimination capability to model high-level semantic information present in RS images (and thus the retrieval performance); and 2) computational complexities associated to retrieval and feature extraction time.
\end{abstract}

Index Terms - local binary pattern, content based image retrieval, remote sensing.

\section{INTRODUCTION}

The rapid development of satellite systems has led to an unprecedented growth of remote sensing (RS) image archives. This raises urgent demands in development of fast and accurate content-based RS image retrieval (CBIR) systems. A CBIR system is generally composed of two main components [1]: 1) an image description component that represents the content of images by a set of features (i.e., image descriptors); and 2) a retrieval component that matches the descriptor of the query image with those of archive images and returns the most similar images to the query image. In the framework of large-scale RS CBIR problems, RS image descriptors should: 1) be discriminative and robust to large intra-category (i.e., class) variations due to the illumination, rotation and scale variations; 2) accurately model the complex semantic image content; and 3) have low computational complexity. Among the several image descriptors (e.g., GIST, Scale Invariant Feature Transform (SIFT)-based image descriptors) Local Binary Pattern (LBP) texture descriptors have attracted significant attention in RS. The standard LBP represents the relationship of each pattern (i.e., pixel) in a given image with its neighbors located on a circle around that pixel by a binary code. Then, a histogram of LBPs is built to describe the images. The success of LBP is due to: 1) its easy implementation; 2) its invariance to monotonic illumination changes; and 3) its low computational complexity [1]. However, the standard LBP has some critical limitations for RS CBIR problems. The quantization process of neighborhood pixels into binary code is a scalar quantization, which is sensitive to noise. Thus, different structural patterns may have the same binary code. Moreover, the LBP captures only the very local structure of the texture and has limitations to detect large-scale textural structures (i.e., it is insufficient to capture macrostructure information). Moreover, the traditional LBP is sensitive to rotation variations, which is not applicable for real RS CBIR problems (where archives consist of images with large rotation variations). In addition, RS images are usually characterized by several image channels that may have a high correlation. However, the standard LBP neglects the correlation among spectral channels (i.e., does not exploit the inter-band information) while describing images.

In the computer vision community, several LBP variants have been developed, which are beneficial to overcome the abovementioned problems in RS CBIR. Accordingly, in this paper we present a study to analyze and compare different LBP variants both theoretically and experimentally under the light of RS CBIR. All the considered algorithms are introduced as a first time in RS. In the paper, we initially present a categorization of the LBP algorithms based on their characterization and summarize the latest developments related to each category in compute vision. In particular, we present, adapt to RS data properties and test LBP variants that can be used with any kind of RS images (e.g. multispectral, hyperspectral, SAR images). The effectiveness of the LBP algorithms is analyzed in terms of: 1) their discrimination capability to model high-level semantic information present in RS images (and thus their capability to lead to high retrieval accuracy); 2) computational time required for their computation; and 3) computational time required for image retrieval (which depends on the length of the LBP descriptors). 


\section{ADVANCED LOCAL BINARY PATTERNS}

Let $\Upsilon=\left\{\mathbf{X}_{1}, \mathbf{X}_{2}, \ldots, \mathbf{X}_{P}\right\}$ be an archive made up of a very large number $P$ of RS images, where $\mathbf{X}_{i}$ is the $i$-th image. To find the most similar images to a query image $\mathbf{X}_{q}\left(\mathbf{X}_{q} \in \Upsilon\right.$ or $\left.\mathbf{X}_{q} \notin \Upsilon\right)$, CBIR systems initially characterize each image $\mathbf{X}_{i}$ with a descriptor. In this paper, we focus our attention on the use of the LBP as a descriptor. LBP associated to a pixel $x_{c}^{i}$ in the image $\mathbf{X}_{i}$ is calculated by comparing its value with those of its $p$ neighboring pixels $\left\{x_{1}^{i}, x_{2}^{i}, \ldots, x_{p}^{i}\right\}$ evenly distributed on a circle of radius $r$ centered on $x_{c}^{i}$ as [1]:

$\operatorname{LBP}\left(x_{c}^{i}\right)=\sum_{n=0}^{p-1} s\left(x_{n}^{i}-x_{c}^{i}\right) 2^{n}$

where $\mathrm{s}()$ is the sign function. The neighbor samples $x_{n}^{i}$ that do not fall exactly in the center of pixels are computed by interpolation. Then, the image is characterized by the probability distribution of the LBP patterns given in $\mathbf{X}_{i}$. The contribution of this paper consists in introducing the LBP variants for large-scale RS retrieval problems to provide high time-efficient (in terms of speed) and accurate search capability within huge RS data archives. To this end, on the basis of the CBIR problems in RS, we categorize the LBP variants into two main categories. 1) single-band specific LBP algorithms, and 2) inter-band correlation sensitive LBP algorithms (see Fig. 1).

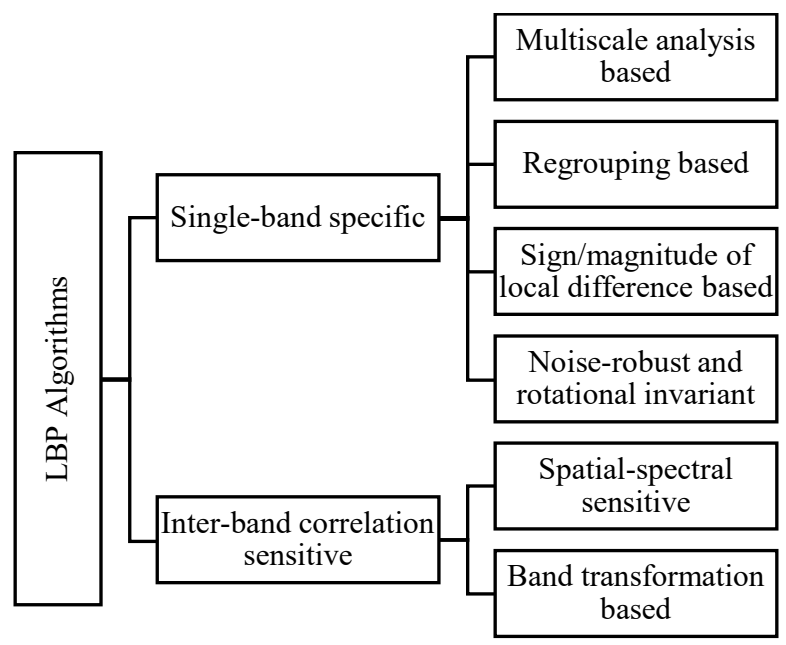

Fig. 1. The categorization of different LBP algorithms

\subsection{Single-band specific LBP algorithms}

The LBP algorithms of this category aim at describing each image band separately from each other. To this end, there are several algorithms developed in computer vision that can be analyzed within four sub-categories: i) multiscale analysis based LBP algorithms (which allow any radius value $r$ with varying number $p$ of samples in the neighborhood); ii) regrouping based LBP algorithms; iii) LBP algorithms that exploit sign and magnitude of local difference; and iv) noiserobust and rotation-invariant LBP algorithms. All these LBP variants allow to control different amount spatial information used to describe the single-band images. With regard to multiscale analysis, Extended LBP (ELBP) [2] and Median Robust Extended LBP (MRELBP) [3] are among the best LBP variants that can accurately describe the local texture. In details, ELBP encodes distinctive spatial relationships in a local region using three LBP-like descriptors [2]. These descriptors capture information from the intensity of the center pixel, its circularly located neighbors and radial differences. MRELBP captures both the micro-textural and macro-textural structures [3]. The macro-texture structure capturing is achieved through replacing individual pixel intensities with patch filter responses. Patches of filters are initially applied to the center pixel and its local neighbors and then the filter responses are taken for the LBP computation. i.e., LBPs are applied on the filter responses rather than pixels intensity values. Other LBP variants of this category are three patch LBP, four patch LBP, pixel to Patch.

LBP regrouping aims at identifying the most informative pattern groups and assigning similar value to all patterns that fall to the same group. Then, then histogram is built based on the grouped LBPs. This approach significantly reduces the feature dimensionality (and thus the required time for image retrieval). There are two different LBP grouping approaches: i) heuristically grouping of LBPs; and ii) discriminative grouping LBPs. Uniform LBP (uLBP) is one of the most effective heuristically regrouping LBP algorithm [1]. Some LBPs occur more frequently than the others, and thus the uLBP method aims to maintain only the uniform patterns (those that most frequently occur), while grouping the other LBPs into non-uniform pattern group. In this method, uniformity $U$ of $x_{c}^{i}$ is measured as [1]:

$U\left(x_{c}^{i}\right)=\sum_{j=1}^{p-1}\left|s\left(x_{\bmod (j, P)}^{i}-x_{c}^{i}\right)-s\left(x_{j-1}^{i}-x_{c}^{i}\right)\right|$

This measure counts the bitwise transitions from 0 to 1 or vice versa. The uniform patterns refer to the samples, whose uniformity values $U$ are less than or equal to 2 . The uniform LBP descriptor has $p(p-1)+3$ categories consisting of $p(p-1)$ +2 distinct uniform patterns and one nonuniform group containing all nonuniform patterns. Concerning the discriminative grouping LPBs, the most discriminative algorithm is the dominant LBPs (DLBPs) that are computed before applying any grouping [11]. In the DLBP, the most frequently occurring LBPs, called dominant patterns, are selected by examining the occurrence frequencies of LBPs (infrequently-occurring LBP groups are discarded).

The third sub-category includes LBP algorithms that exploit sign and magnitude of local difference. In [4], it is shown that the sign component is more important than the magnitude 
Table 1. Average recall and computational time required for feature extraction (FE) and image retrieval (in seconds) associated to different LBP algorithms

\begin{tabular}{|c|c|c|c|c|c|c|c|c|c|c|}
\hline \multirow{2}{*}{ Results } & \multirow{2}{*}{$\begin{array}{l}\text { Standard } \\
\text { LBP }\end{array}$} & \multicolumn{6}{|c|}{ Single-band specific LBPs } & \multicolumn{3}{|c|}{ Inter-band correlation sensitive LBPs } \\
\hline & & ELBP & CLBP & uLBP & DLBP & riLBP & MRELBP & LBP-TOP & 3D-LBP & maLBP \\
\hline $\begin{array}{c}\begin{array}{c}\text { Recall } \\
(\%)\end{array} \\
\end{array}$ & 69.87 & 76.10 & 69.87 & 68.82 & 64.53 & 67.04 & 76.17 & 70.89 & 69.08 & 70.21 \\
\hline FE time & 0.06 & 0.29 & 0.07 & 0.12 & 0.03 & 0.14 & 0.29 & 0.52 & 0.60 & 6.64 \\
\hline $\begin{array}{c}\text { Retrieval } \\
\text { time }\end{array}$ & 74.58 & 60.72 & 76.36 & 24.49 & 24.72 & 23.62 & 62.25 & 23.75 & 43.03 & 97.55 \\
\hline
\end{tabular}

component in preserving the local difference information. As an example, algorithm for this category is the completed LBP (CLBP) [4] that exploits the two-complementary information (magnitude and sign) using two texture operators. The first descriptor represents the sign, which equals to the traditional LBP, while the second descriptor represents the magnitude (local variance) of the texture.

The LBP algorithms of last sub-category, which are noiserobust and rotation-invariant LBP algorithms, aim to apply filtering on the image as a pre-processing operation prior to LBP computation. For example, LBP variants like LGBP [5] uses Gabor filtering, since Gabor filters are complementary to the LBP. LBPs capture information in small scale area whereas Gabor filters capture large-scale details. Other LBP variants like MRELBP [3] exploits median filter, achieving a noise-robust image descriptor. The rotation invariant LBP algorithms aim to remove the effect of rotation in the characterization of the images. As an example of this category is the rotation invariant LBP (riLBP). To reduce the feature dimensionality, rotation invariant uniform LBP is also presented in [1].

It is worth noting that all the LBP variants of this category is applied to each image band separately and then the histogram is built to describe each image band. Then, the histograms of bands are simply concatenated to describe the image.

\subsection{Inter-band correlation sensitive LBP algorithms}

The LBP algorithms of this category aim at characterizing the inter band correlation while extracting the LBP descriptor of an image. For high dimensional RS images, as mentioned before, the simple concatenation approach of the histograms for each image band does not model the existing inter-band correlation. To address these problems, several LBP algorithms that extract and exploit the inter-channel information in the process of LBP computation are developed in computer vision. We divide these algorithms into two subcategories: 1) spatial and spectral information sensitive LBP algorithms, and 2) band-transformation based LBPs. Volume LBP (VLBP) [6], three orthogonal planes LBP (LBP-TOP) [12] and 3 dimensional-LBP (3D-LBP) [7] are techniques of the category of the spatial and spectral information sensitive
LBP algorithms. The VLBP combines the motion and appearance to describe dynamic textures [6], and can be adopted to RS image characterization by replacing the temporal domain with the spectral domain. For a pixel given an image band, circularly symmetric volume neighbor sets are extracted from successive image bands. This approach is computationally demanding particularly for very high dimensional RS images. To alleviate this problem, the LBPTOP is developed, which exploits the co-occurrences on three orthogonal planes. In 3D-LBP [7], an RS image descriptor can be obtained by reconstructing the image pixel as a 3D data cube considering the spatial and spectral coordinates. Then, the standard LBP has been extended into 3-D LBP model through forming a 3D regular octahedral frame to characterize the spectral-spatial relationship.

mCENTRIST [8] and Multichannel Decoded LBP [9] are examples of the band-transformation based LBPs. In details, two multichannel decoded local binary pattern approaches are proposed in [9] for the combination of the LBPs extracted from more than one image band. These methods, namely multichannel adder based local binary pattern (maLBP) and multichannel decoder based local binary pattern (mdLBP), exploit the local binary pattern information of multiple image bands in efficient manners. This is achieved by modeling the local information of multiple image channels on the basis of the adder and decoder concepts, respectively, before the computation of the histogram.

\section{EXPERIMENTAL RESULTS}

To evaluate the effectiveness of the various LBP variants, we carried out experiments on a benchmark archive (which consists of 2100 images selected from aerial orthoimagery with a spatial resolution of $30 \mathrm{~cm} \mathrm{[10]).} \mathrm{In} \mathrm{the} \mathrm{experiments,}$ we considered several algorithms from two main LBP categories, which are promising in computer vision but never used in the framework of RS CBIR. From the single-band specific LBP algorithms, the ELBP, the CLBP, the uLBP, the riLBP, the MRELBP and the DLBP were selected. From the category of inter-band correlation sensitive LBP algorithms, the LBP-TOP, the 3D-LBP and the maLBP algorithms were 
chosen. In the retrieval phase, $k$-nearest algorithm was used, i.e., similarities between the descriptors of the query image and those of all archive images were estimated and images related to the lowest distance were retrieved. The retrieval performance was assessed on top-20 retrieved images (i.e., $k=20$ ). As a distance measure, Chi-square distance was considered. Results associated to each LBP descriptor are provided in terms of: i) average recall; ii) average feature extraction time; and iii) average retrieval time obtained in 2100 trials performed with 2100 selected query images from the archive. All the experiments are implemented via MATLAB ${ }^{\circledR}$ on a standard PC with Intel ${ }^{\circledR}$ Xeon ${ }^{\circledR}$ CPU E51650v2@3.50GHz,16GB RAM.

Table 1 shows the average recall obtained by the considered LBP algorithms and the related computational time required for feature extraction and retrieval processes. By analyzing the table, one can see that all the LBP variants of the two categories result in higher retrieval accuracies than the standard LBP. As an example, the ELBP and the MRELBP yield almost 7\% higher recall than the standard LBP with reduced retrieval time at the cost of increased feature extraction time. It is worth noting that the retrieval time reduction is due to reduced feature dimensionality. Concerning the retrieval time of the LBP algorithms, from the table it can be seen that the grouping based LBP algorithms (e.g., the uLBP and the DLBP) are very fast, since they represent images with low-dimensional descriptors. However, they provide lower recall with respect to the standard LBP. Thus, these algorithms are preferable when the computational time is more important than the retrieval accuracy and vice-versa. The highest recall is obtained by the MRELBP (which is robust to noise and rotation invariant) with the cost of increased time for the feature extraction. However, the retrieval time required for this descriptor is slightly lower than the standard LBP. Inter-band correlation sensitive LBP algorithms provide similar recall with respect to the algorithms of the other two categories. It is worth noting that the images in the considered benchmark archive consist of three channels only. We expect that for real RS CBIR problems where images are high dimensional the effectiveness of the inter-band correlation sensitive LBP algorithms will be much more relevant.

\section{CONCLUSION}

In this paper, we have presented a comparative study of LBP variants for RS image retrieval problems. In particular, we have grouped the LBP variants into two main categories: 1) single-band specific LBP descriptors; and 2) inter-band correlation sensitive LBP algorithms. The considered LBP variants have been considered for the first time in the framework of RS CBIR problems. The theoretical and experimental analysis show that for RS CBIR problems LBP algorithms should be chosen according to the dimension of the considered RS images and also the desired tradeoff between the retrieval accuracy and the computational time.

\section{ACKNOWLEDGEMENT}

This work was supported by the European Research Council under the ERC Starting Grant BigEarth-759764.

\section{REFERENCES}

[1] T. Ojala, M. Pietikainen and T. Maenpaa, "Multiresolution grayscale and rotation invariant texture classification with local binary patterns", IEEE Transactions on Pattern Analysis and Machine Intelligence, vol. 24, no. 7, pp. 971-987, 2002.

[2] L. Liu, L. Zhao, Y. Long, G. Kuang, and P. Fieguth, "Extended local binary patterns for texture classification", Image Vis. Comput., vol. 30, no. 2, pp. 86-99, Feb. 2012.

[3] L. Liu, S. Lao, P. W. Fieguth, Y. Guo, X. Wang, M. Pietikäinen, "Median robust extended local binary pattern for texture classification", IEEE Transactions on Image Processing, vol. 25, no. 3, pp. 1368-1381, 2016.

[4] Z. Guo, L. Zhang, D. Zhang, "A completed modeling of local binary pattern operator for texture classification", IEEE Transactions on Image Processing, vol. 9, no.16, pp. 1657-1663, 2010 .

[5] W. Zhang, S. Shan, W. Gao, X. Chen, H. Zhang, "Local Gabor binary pattern histogram sequence: a novel non-statistical model for face representation and recognition", IEEE International Conference on Computer Vision (ICCV), pp. 786-791, 2005.

[6] G. Zhao, and M. Pietikainen, "Dynamic texture recognition using local binary patterns with an application to facial expressions", IEEE Transactions on Pattern Analysis and Machine Intelligence, vol. 29, no. 6, pp. 915-928, 2007.

[7] S. Jia, J. Hu, J. Zhu, X. Jia, Q. Li, “Three-dimensional local binary patterns for hyperspectral imagery classification", IEEE Transactions on Geoscience and Remote Sensing, vol. 55, no. 4, pp. 2399-2413, 2017.

[8] Y. Xiao, J. Wu, J. Yuan, "mCENTRIST: A multi-channel feature generation mechanism for scene categorization", IEEE Transactions on Image Processing, vol. 23, no. 2,pp. 823-836, 2014.

[9] S. R. Dubey, S. K. Singh, R. K. Singh, "Multichannel decoded local binary patterns for content-based image retrieval", IEEE Transactions on Image Processing, vol. 25, no. 9, pp. 4018-4032, 2016.

[10] Y. Yang, and S. Newsam, "Geographic image retrieval using local invariant features", IEEE Transactions on Geoscience and Remote Sensing, vol. 51, no. 2, pp. 818-832, 2013.

[11] S. Liao, M. W. K. Law, A. C. S. Chung, "Dominant Local Binary Patterns for Texture Classification", IEEE Transactions on Image Processing, vol. 18, no. 5,pp. 1107-1118, 2009.

[12] T. R. Almaev and M. F. Valstar, "Local gabor binary patterns from three orthogonal planes for automatic facial expression recognition", Humaine Association Conference on Affective Computing and Intelligent Interaction, pp. 356-361, 2013. 